\title{
Liver Transplant as Treatment Modality for Inflammatory Myofibroblastic Tumor of the Liver
}

\author{
Aniruddh Setya ${ }^{1 *}$, Jaswinder Kaur ${ }^{2}$, Anand Gupta ${ }^{2}$ and Nishant Wadhwa ${ }^{2}$ \\ ${ }^{1}$ Department of Pediatric Gastroenterology, Hepatology and Nutrition, University of Florida, USA \\ ${ }^{2}$ Department of Pediatric Gastroenterology, Hepatology and Nutrition, Sir Ganga Ram Hospital, India
}

*Corresponding author: Aniruddh Setya, Department of Pediatric Gastroenterology,

Hepatology and Nutrition, University of Florida, Gainesville, USA.

Received Date: March 02, 2020

Published Date: March 12, 2020

\section{Case Report}

A 3-year-old African female child with a noncontributory birth, past and family history presented with progressively increasing abdominal distension for 3 months associated with intermittent fever, abdominal pain and cough. On her initial evaluation, contrastenhanced computed tomography (CECT) abdomen revealed a mass in the right hepatic lobe with thrombus in the inferior vena cava (IVC) and right ventricle with enlarged aortocaval, para aortic and celiac lymph nodes with mild ascites. Liver biopsy was deferred, as the patient was sick upon presentation. Suspecting it to be hepatoblastoma, she was given 4 cycles of Doxorubicin and Carboplatin. Tumor markers carcinoembryonic antigen (CEA), Beta human chorionic gonadotropin (hCG) and Alpha-fetoprotein (AFP) were found to be negative. In view of poor response to chemotherapy and persistent symptoms, she was brought to our hospital in
New Delhi, India. On presentation, she was hemodynamically stable and had firm hepatomegaly and pallor. Positron emission tomography-computed tomography (PET CT) abdomen revealed hypertrophied left lobe of the liver, 18F-fluorodeoxyglucose (FDG) avid large heterogenous arterial enhancing lesion in the right lobe liver involving segment VII, VIII, VI with extension into IVC, right atrium and ventricle. There was bilateral moderate pleural effusion with atelectasis. She underwent an ultrasound guided biopsy of the lesion which was suggestive of inflammatory Myofibroblastic tumor (bundles of oval to spindle shaped fibroblastic cells in a collagenized stroma with cells showing mild pleomorphism). Immunohistochemical stain for Anaplastic Lymphoma Kinase (ALK) was positive but stain for SMA, CD34 and CD31 were negative. Crizotinib (ALK inhibitor) was started for reduction of the tumor volume and patient improved.

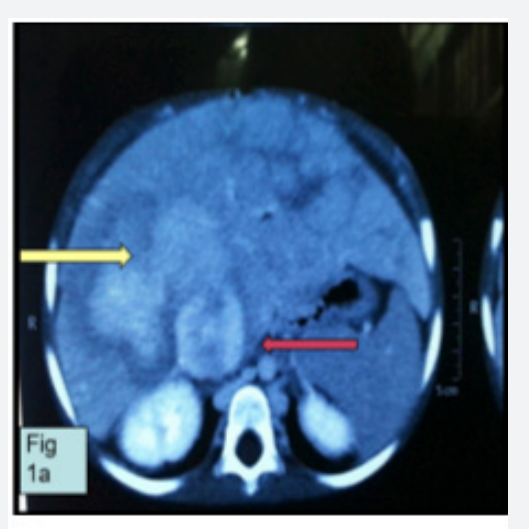

Figure 1a: Contrast enhanced Computed tomography scan showing relatively well defined heterogeneously enhancing lobulated lesion. It is predominantly involving the right lobe of liver (segment 8) and is seen extending into IVC*. 


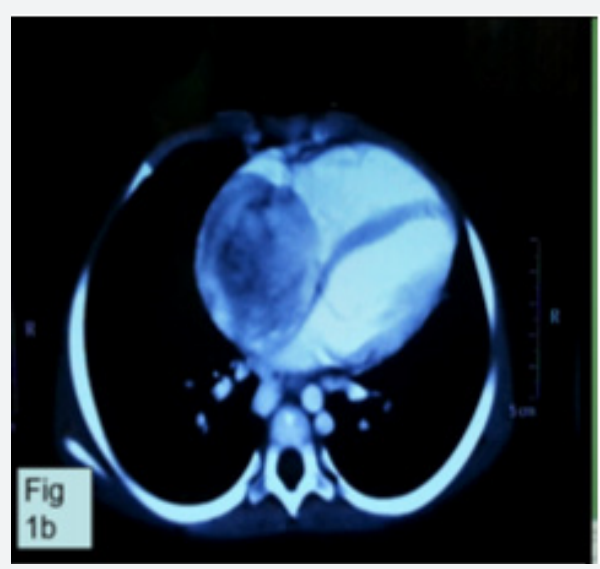

Figure 1b: The lesion is extending in the right atrium up to the right AV groove. Radio imaging suggestive of aggressive malignancy.

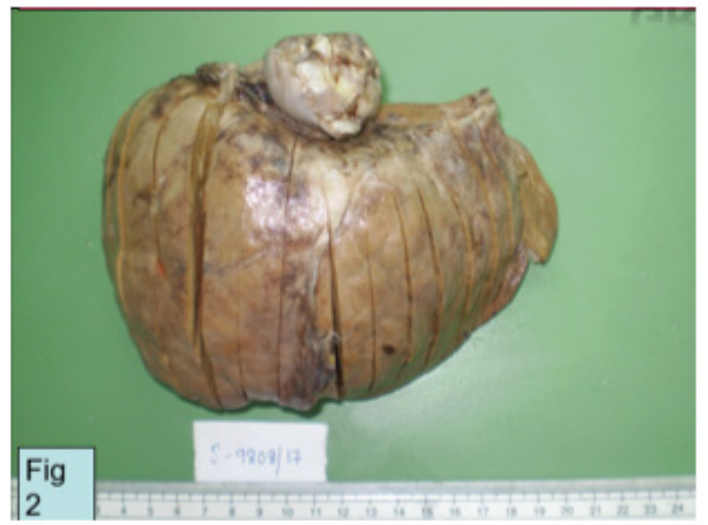

Figure 2: Gross examination shows an explant liver which is markedly enlarged in size. The hilar aspect shows a dilated segment of IVC, The lumen of which is filled with tumor which is protruding beyond the cut margin.

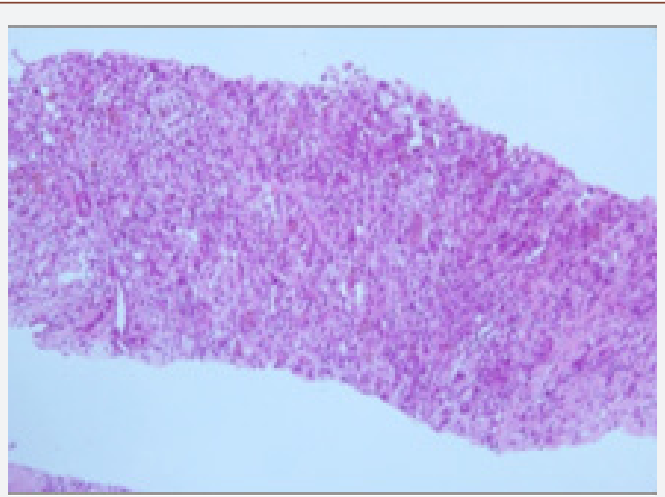

Figure 3: Core liver biopsy shows a lesion composed of cytologically bland spindle cells and prominent inflammatory infiltrate (H\&E 20X). lumen of which is filled with tumor which is protruding beyond the cut margin.

3 months later ultrasound doppler revealed a lobulated hypoechoic mass involving the supra hepatic IVC $(7.8 \times 4.3 \times 4 \mathrm{~cm})$ extending into the right atrium. Echocardiogram showed a localized mass in the RA filling the whole right atrial cavity and obstructing the IVC flow. Left ventricle ejection fraction (LVEF) was $60 \%$ with normal right and left ventricle. Mitral valve was normal with a mild tricuspid regurgitation and normal coronary vessels. These findings were confirmed on CT angiography. As the hepatic tumor was non-respectable along with secondary cirrhotic changes in the liver, a living donor liver transplant with excision of the RA wall, IVC and liver was planned. Pre-transplant investigations revealed an elevated CRP (due to the inflammatory nature of the tumor) despite a negative sepsis workup. Intra-op findings revealed a hard, lobulated externally palpable mass, totally obliterating the IVC and $90 \%$ of the right atrium (RA)volume. Tumor was excised along with the wide rim of RA and the IVC was excised from the RA. Posterior 
wall of the RA was reconstructed using autologous pericardium and a Y configured PTFE graft was used. The liver was explanted with the involved IVC, posterior limb of the IVC graft was anastomosed to the supra renal IVC. The donor liver was then transplanted, vascular anastomosis done, and adequate flows were confirmed. Post- operatively she was admitted in pediatric critical care unit for 13 days, suffered no complications and was subsequently discharged on immunosuppression therapy. On follow up one month and six months post-transplant was found to be doing well (Figures $2 \& 3$ ).

\section{Discussion}

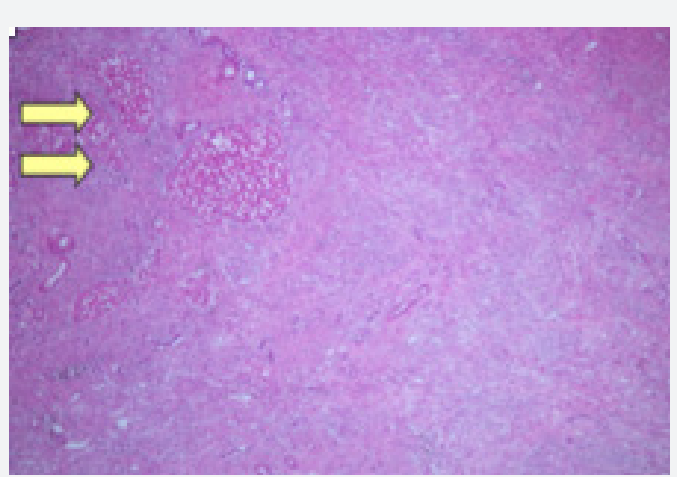

Figure 4: Section from the hepatectomy specimen showing a spindle cell lesion arranged in fascicles and storiform pattern normal liver parenchyma is seen

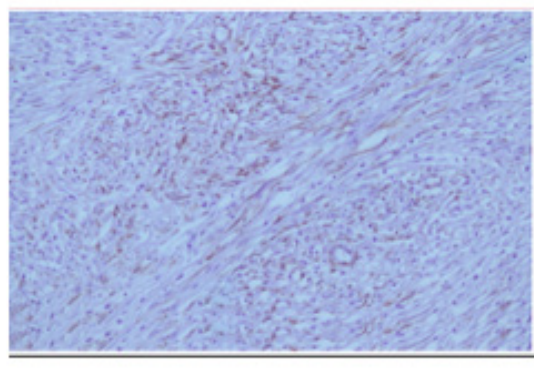

Figure 5: Scattered cells show positivity for SMA.

Inflammatory Myofibroblastic tumors (IMT) are rare tumors and are often referred under the umbrella term of Inflammatory Pseudotumor (IPT). The concept of IMTs as neoplasms was established with the discovery of cytogenetic aberrations and the subsequent recognition of anaplastic lymphoma tyrosine kinase (ALK) gene rearrangements as a recurrent aberration in IMTs. The World Health Organization (WHO) classification currently defines an IMT as an intermediate soft-tissue tumor comprising spindle cells that exhibit myofibroblast differentiation with numerous inflammatory cells, plasma cells, and/or lymphocytes [1]. They commonly occur in liver or lungs [2-4]. These tumors are difficult to distinguish from other neoplasms based on radiographic imaging alone and histologic analysis is required to establish the diagnosis. Recently, Tang, et al. [5] analyzed 64 cases diagnosed as IMT of the liver and observed that they had similar age and gender distributions as IPT. The most common clinical presentation was abdominal pain followed by fever and no other symptoms. He also observed that both in IPT and IMT of the liver, laboratory investigations often suggest an inflammatory process: leukocytosis, neutrophilia, elevated erythrocyte sedimentation rate (ESR) and C-reactive protein (CRP), anemia, thrombocytosis, polyclonal hypergammaglobulinemia, and slightly elevated liver enzymes. Tumor markers such as serum alpha-fetoprotein (AFP) and carcinoembryonic antigen (CEA) were always normal, although cancer antigen 19-9 (CA 199) was raised in some patients. The same was true for our patient who showed an elevated CRP and liver enzymes at presentation. Management of IMT is still controversial. A retrospective review of pediatric patients diagnosed with IMFT over a 21 year period at two tertiary centers found that IMFT is a rare neoplasm which has a relatively benign course with an overall survival of over $90 \%$. When completely resected with microscopically clear margins, IMFT can be curative, making surgical resection the mainstay of therapy [6]. It is also postulated that when an IMFT is suspected, cross-sectional imaging (CT or MRI depending on organ system involved) is the most beneficial for operative planning. Some authors have advocated a conservative approach. However, the natural course of these tumors without intervention is not fully known. Importantly, when recurrence occurs, it is usually in cases with incomplete resection where no chemotherapy or radiotherapy was provided. Therefore, it is suggested that surgical resection should be recommended for 
all lesions if not prohibited by anatomic location or morbidity [7]. It has been proposed that in case of an unresectable and metastatic ALK-expressing IMT, ALK inhibitors may provide therapeutic benefit and durable responses may be achieved [8]. Our patient was started on Crizotinib and showed some response with a shrinking tumor size but despite three months of therapy her tumor persisted. We believe, to the best of our knowledge that liver transplant in a patient with diagnosed IMT has not been used as a modality for treatment in the past and our case was unique in terms of the extent of the tumor which made it non respectable (Figures 4, 5 \& 6).

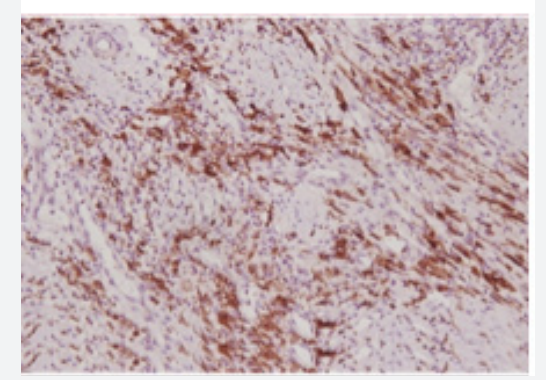

Figure 6: Tumor positive for ALK 1.

\section{Conclusion}

IMT is a rare neoplasm of children with abdomen and pelvis a frequent presenting site. There are no standardized protocols or guidelines, but we believe that early aggressive treatment and prompt biopsy and/or excision is the key to successful management. In a scenario where the tumor is non respectable liver transplant is a viable treatment modality. Need of the hour is to standardize diagnostic and treatment protocol for managing IMT in pediatrics.

\section{Acknowledgement}

All authors equally contributed to this paper with data collection, literature review and analysis, drafting and critical revision and editing, and final approval of the final version.

\section{Conflict of Interest}

No conflict of interest.

\section{References}

1. Coffin CM, Hornick JL, Fletcher CD (2007) Inflammatory myofibroblastic tumor: comparison of clinicopathologic, histologic, and immunohistochemical features including ALK expression in atypical and aggressive cases. Am J Surg Pathol 31: 509-520.

2. Siminovich M, Galluzzo L, Lopez J (2012) Inflammatory myofibroblastic tumor of the lung in children: anaplastic lymphoma kinase (ALK) expression and clinicopathological correlation. Pediatr Dev Pathol 15(3): 179-186.

3. Yu DC, Grabowski MJ, Kozakewich HP, Perez-Atayde AR, Voss SD, et al. (2010) Primary lung tumors in children and adolescents: a 90-year experience. J Pediatr Surg 45(6): 1090-1095.

4. Chable-Montero F, Angeles-Angeles A, Albores-Saavedra J (2012) Inflammatorymyofibroblastic tumor of the liver. Ann Hepatol 11(5): 708-709.

5. Tang L, Lai ECH, Cong W, Li A, Fu S, et al. (2010) Inflammatory myofibroblastic tumor of the liver: a cohort study. World J Surg 34: 309313.

6. Dalton BG, Thomas PG, Sharp NE, Manalang MA, Fisher JE, et al. (2016) Inflammatory myofibroblastic tumors in children. Journal of pediatric surgery 51: 541-544.

7. Golsmith PJ, Loganathan A, Jacob M, Ahmad N, Toogood GJ, et al. (2009) Inflammatory pseudotumors of the liver: a spectrum of presentation and management options. Eur J Surg Oncol 35: 1295-1298.

8. Tothova Z, Wagner AJ (2012) Anaplastic lymphoma kinase-directed therapy in inflammatory myofibroblastic tumors. Curr Opin Oncol 24: 409-413. 